\section{SPSSCONV: a program to convert SPSS system files into OSIRIS datasets}

\author{
JAMES P. McGREGOR \\ Department of Political Science \\ The Ohio State University, Columbus, Ohio 43210
}

SPSSCONV is designed to complement the OSIRIS-SPSS interface available in the more recent versions of SPSS. The program accepts a standard SPSS system file and produces OSIRIS dictionary and data files as output. In its present form SPSSCONV requires no control cards.

Input. Input for SPSSCONV is a standard SPSS system file.

Output. Two output files are written on a medium of the user's choice. The first file is a standard OSIRIS $t$-card dictionary constructed from information contained in the SPSS dictionary. The second file is a standard OSIRIS data file. Printed output from the program consists of the SPSS file descriptor information (file name, date of creation, number of variables) and a cross-reference table showing the equivalence between the SPSS and OSIRIS dictionaries.

Restrictions. Due to conceptual incompatabilities between SPSS and OSIRIS, a number of compromises were necessary, the most important of which will be noted here. (1) All values in the SPSS file are converted to decimal values with a field width of eight and a precision of three places to the right of the decimal. (2) Only the first SPSS missing data code is transferred to the OSIRIS dictionary. (3) Variable labels are truncated to 24 characters in the OSIRIS dictionary.

Computer and Language. SPSSCONV was written in $\mathrm{PL} / \mathrm{I}$ (IBM Optimizing Compiler) for the IBM 370/165.

Availability: A source deck and program write-up for SPSSCONV can be obtained from the computer program archive of the Behavioral Science Laboratory, 404B West 17th Avenue, The Ohio State University, Columbus, Ohio 43210 for duplication costs. Requests should be sent directly to the Behavioral Science Laboratory.

\section{SPSSMAT: A program to convert OSIRIS correlation matrices to SPSS format}

\author{
JAMES P. MCGREGOR \\ Department of Political Science \\ The Ohio State University, Columbus, Ohio 43210
}

The SPSS package is severely limited with respect to the types of nonparametric correlation coefficients available to routines which accept matrix input (e.g., REGRESSION, PARTIALS, FACTOR, etc.). Additionally, the largest nonparametric matrix capable of being produced by the current version of SPSS (Version 6) is 30 by 30 and is too small for many purposes. The OSIRIS package is much less limited in these respects and is capable of producing matrices of exotic coefficients of large size. The format of OSIRIS matrices, however, is incompatible with the SPSS system. SPSSMAT was designed to make the necessary adjustments to render OSIRIS correlation matrices usable in SPSS.

Input. Input consists of a program control card and an OSIRIS correlation matrix which may or may not include variable means and standard deviation. The control card informs the program of the presence or absence of means and standard deviations and allows program output to be tailored to the user's needs.

Output. Two data files and a program report are output from the program. OSIRIS variable labels can be converted to SPSS format and an SPSS VARIABLE LIST card produced on a file separate from the converted matrix. This feature allows the user to store large matrices on tape or disk and punch the SPSS VARIABLE LIST and VAR LABELS cards. The second output file is the converted matrix and means and standard deviations (the latter are optional and may be suppressed). A program report consisting of control card parameter interpretation and size of the converted matrix is also output.

Computer and language. SPSSMAT was written in FORTRAN IV (G1) for the IBM 370/165.

Availability. A source deck and program write-up for SPSSMAT can be obtained from the computer program archive of the Behavioral Science Laboratory, 404B West 17th Avenue, The Ohio State University, Columbus, Ohio 43210 for duplication costs. Requests should be sent directly to the Behavioral Science L,aboratory. 